\title{
\%
}

\section{RESPONSABILIDAD SOCIAL CORPORATIVA Y CÓDIGO DE BUEN GOBIERNO Cumplimiento por las empresas del IBEX}

El Código de buen gobierno (CBG) de las sociedades cotizadas de 2015 es el primero, en nuestro país, que incluye recomendaciones específicas en materia de responsabilidad social corporativa (RSC), apostando definitivamente por un modelo stakeholders o pluralista de la empresa.

En este trabajo se analiza el nivel de cumplimiento de dichas recomendaciones por parte de las empresas del IBEX durante los dos primeros años de vigencia de dicho código, concluyéndose que éste es elevado y su evolución, positiva. Se destaca la rapidez con la que se ha producido la incorporación de la RSC en el gobierno corporativo (GC) de estas empresas.

Palabras Clave: Código de buen gobierno, gobierno corporativo, responsabilidad social corporativa, IBEX. Clasificación JEL: G34, M14.

\section{Introducción}

A principios del presente siglo se produjeron una serie de escándalos empresariales y financieros $^{1}$ que evidenciaron que la crisis no fue motivada sólo por fuertes desequilibrios estructurales, sino también por una determinada

\footnotetext{
*Universidad de Almería.

**Universidad Internacional de La Rioja.

Versión de septiembre de 2017.

1 Enron, una de las principales empresas americanas, designada por la revista Forbes varios años consecutivos como la más innovadora, se declaraba en quiebra en el 2001, tras protagonizar el mayor fraude empresarial conocido hasta entonces. En 2008 se produce la quiebra de Lheman Brothers, cuarto banco de inversión de Wall Street, víctima del monstruo que contribuyó a crear en el negocio de las hipotecas subprime. En 2010, British Petroleum ocasiona el mayor desastre ecológico de la historia.
}

cultura de management corporativo basada en la creación de valor para el accionista y la alta dirección.

Ello ocasionó un aumento en la preocupación de la sociedad en general por determinadas prácticas empresariales y, con ello, la necesidad de introducir cambios y regulaciones para evitar situaciones similares en el futuro. Empezó a considerarse que las empresas no son entes exclusivamente privados, ya que su actuación tiene impactos decisivos en el empleo, el consumo, el medioambiente y la desigualdad social. Fue adquiriendo mayor importancia la ética empresarial y los valores de la RSC, iniciándose un proceso de $D$ 
convergencia de dos términos, GC y RSC, que, tradicionalmente, estaban separados (Alejos, 2015).

EI GC nace al calor de la teoría de la agencia, según la cual las sociedades cotizadas se configuran en torno a un entramado de relaciones entre los propietarios y los directivos (Lizcano, 2006). En dicho entramado de relaciones pueden suscitarse conflictos de intereses, derivados del hecho de que los directivos disponen de la información, los medios y la oportunidad para lograr maximizar las ganancias de la empresa en beneficio propio o de un grupo reducido, en detrimento de los intereses del resto de los interesados (Fushimi, 2011). Son los denominados «problemas de agencia» que se generan por el escaso peso de los accionistas en la toma de decisiones de la empresa, dada su extrema atomización; por el conflicto de intereses que puede surgir entre accionistas mayoritarios y minoritarios, y por el conflicto de intereses entre accionistas y acreedores.

Un buen gobierno corporativo (BGC) es aquel que procura evitar o minimizar los problemas de agencia, a través de diversos métodos y procedimientos que tienden a garantizar que no existan los elementos que los generan (Fushimi, 2011).

Siguiendo a Lizcano, el BGC sería aquel capaz de canalizar adecuadamente el entramado de relaciones entre los propietarios y los directivos de las empresas, en el campo de la organización, gestión y control empresarial con el fin de alcanzar los objetivos fijados (Lizcano, 2006).

Los CBGC aparecen como herramientas de ayuda a las empresas para implementar políticas de BGC. Son pronunciamientos en torno al GC, que incluyen recomendaciones precisas, no vinculantes para las empresas, elaboradas por comisiones especializadas de expertos a instancias de organismos oficiales (Lizcano, 2006). Tienen como finalidad establecer criterios y pautas a los que se someterán las empresas, así como recomendaciones sobre transparencia, composición y funcionamiento de órganos de gobierno y relación con los grupos de interés (Alejos, 2015). La mayoría de ellos tienen en común el objetivo de fortalecer la reputación corporativa de las empresas, dotándolas de mayor transparencia y de mayor control interno y externo, con el fin de recuperar la confianza del inversor y de protegerlo, evitando que decisiones basadas en el beneficio a corto plazo puedan minar la salud y el valor futuro de la empresa (Rivero, 2005).

Por otra parte, la RSC representa una forma de entender la empresa desde unos valores y fines específicos que buscan generar valor para todas las partes interesadas en la marcha de aquella. Centra su atención en la satisfacción de las necesidades de los grupos de interés, va más allá del mero cumplimiento de la normativa legal establecida y de la obtención de resultados exclusivamente económicos a corto plazo, supone un planteamiento de tipo estratégico que afecta a la toma de decisiones y a las operaciones de toda la organización, creando valor en el largo plazo y contribuyendo significativamente a la obtención de ventajas competitivas duraderas (Lizcano y Moneva, 2004).

En el año 2015 se aprobó en España el CBG de las sociedades anónimas cotizadas, siendo el primero, de los elaborados en nuestro país, en incluir en su texto recomendaciones específicas en materia de RSC.

El objetivo de este trabajo es analizar el nivel de cumplimiento de las recomendaciones sobre RSC contenidas en el mencionado código por parte de las empresas del IBEX-35. $D$ 
Previamente, analizaremos los distintos enfoques o modelos de GC y su relación con la RSC, haciendo un breve recorrido de los diferentes CBGC que han estado vigentes en nuestro país y las referencias de cada uno de ellos a la RSC.

\section{Gobierno corporativo y responsabilidad social corporativa}

Existen numerosos estudios que evidencian la relación de complementariedad existente entre el GC y la RSC. Un BGC es la base para una sólida RSC. Por otra parte, el GC no puede ser efectivo sin un concepto de RSC (Jamali, Safieddine y Rabbath, 2008).

Podemos definir, siguiendo a Lizcano, tres modelos de GC en función de los fines (misión) de la empresa (Lizcano, 2006).

\section{Modelo shareholder o accionarial}

Los fines de la empresa son únicamente financieros. La empresa es un instrumento para que los accionistas maximicen sus beneficios. Un BGC en este modelo sería aquel que consiguiera maximizar el valor para el accionista.

\section{Modelo stakeholders o pluralista}

El fin de la empresa no se fija exclusivamente en el beneficio para los accionistas, sino que tiene en cuenta también los intereses del resto de los grupos interesados (empleados, clientes, proveedores, Administración Pública, competencia y generaciones futuras). En este modelo un BGC sería aquel que alcance, de manera eficaz, dicho objetivo socialmente responsable.

\section{Modelo accionarial matizado}

Se corresponde con una visión instrumental del modelo stakeholders. La atención a los intereses de los distintos grupos interesados se justifica no desde una perspectiva ética o moral, sino por los efectos positivos que pueda tener sobre la generación de valor para el accionista.

Aunque tradicionalmente el enfoque de las políticas de GC se ha centrado en la defensa de los intereses de los accionistas, con un encuadre claramente accionarial de la empresa, en las últimas décadas estamos asistiendo a un cambio de enfoque, pasando a un modelo pluralista o stakeholders. Dicho cambio se está produciendo tanto por razones instrumentales (modelo accionarial matizado) como por razones éticas o morales (modelo stakeholders). Ello se ha traducido en una mayor presencia de los principios de la RSC en el GC de las empresas.

Esta incorporación de la RSC en el GC no ha estado exenta de dificultades, ya que ha supuesto un importante cambio en el papel tradicional del Consejo de Administración y de los propios administradores de las empresas (mayor diversidad e independencia del Consejo de Administración; incorporación, en el seno del Consejo de Administración, de una comisión específica sobre RSC; mayor transparencia en la composición y funcionamiento de los órganos de gobierno y relación con los grupos de interés; redacción de códigos de conducta; elaboración de informes como medio para lograr la transparencia, etcétera).

$\mathrm{Ha}$ ayudado a este cambio de enfoque la proliferación de los CBGC, en la medida en que han venido incluyendo recomendaciones sobre la diligencia y lealtad de los administradores, así como sobre la necesidad de $\triangleright$ 
transparencia, veracidad y relevancia en las informaciones de la empresa. De esta forma, los CBGC se han convertido en una herramienta de ayuda a la incorporación de la RSC al GC de las empresas.

\section{Códigos de buen gobierno corporativo en España}

Desde finales del pasado siglo se viene produciendo, en sectores empresariales y amplios grupos de la opinión pública, una gran demanda, exigiendo una mayor eficacia, agilidad, responsabilidad y transparencia en el gobierno de las sociedades. Dicha demanda tenía como objetivo fundamental conseguir una mayor credibilidad y una mejor defensa de los intereses de los accionistas. Como respuesta a la misma, comienzan a proliferar los CBGC, que incluyen una serie de criterios y pautas sobre la composición y funcionamiento de los órganos de gobierno de las empresas.

Tradicionalmente, estos informes o códigos se centraron en la defensa de los intereses de los accionistas, con un enfoque claramente accionarial de la empresa. No obstante, con el paso del tiempo, algunos de ellos empiezan a concebir la empresa con un enfoque accionarial matizado, en el que, como se ha dicho, la atención a los stakeholders se justifica por su efecto positivo sobre la generación de valor para el accionista (Lizcano, 2006).

Algunos ejemplos de estos informes, en el contexto internacional, son los siguientes (Ferruz, Marco y Acero, 2010): el informe Treadway ${ }^{2}$ (Committee of Sponsoring Organizations of the

\footnotetext{
2 El informe Treadway de 1987, en Estados Unidos, fue el primero en recomendar la necesidad de constituir una Comisión de Auditoría dentro de los Consejos de Administración de las sociedades como medio para controlar el fraude.
}

Treadway Commission, 1987); el informe Cadbury ${ }^{3}$ (Cadbury, 1992); el informe Hampel ${ }^{4}$ (Hampel, 1998); el informe Vienot ${ }^{5}$ (Vienot, 1995); el informe Winter ${ }^{6}$ (Comisión Europea, 2001b); Principios de la OCDE (Organización para la Cooperación y Desarrollo Económico) sobre GC7 (OCDE, 2004).

Fue, fundamentalmente, a raíz de la publicación de este último, cuando se opta, en muchos códigos, por un enfoque claramente pluralista de la empresa, donde la función principal del GC es la generación de valor para los stakeholders.

Desde la perspectiva de los tres enfoques o modelos analizados en el apartado anterior, a continuación llevaremos a cabo un breve recorrido histórico de los diferentes CBGC que han estado vigentes en nuestro país.

\section{Código Olivencia}

Se aprueba en 1998 como un código ético de buen gobierno, de asunción voluntaria por las $\triangleright$

\footnotetext{
3 El Informe Cadbury de 1992, del Reino Unido, hace una referencia especial a las remuneraciones de los directivos.

4 El Informe Hampel de 1998 establece la necesidad de que existan en las sociedades consejeros independientes, división de tareas entre el presidente y el primer ejecutivo de la compañía, control de las remuneraciones del Consejo, obligación de ofrecer una información financiera equilibrada y comprensible, creación de comités de auditoría y potenciación de las juntas generales de accionistas.

5 El informe Vienot de 1995 se aplica a las sociedades cotizadas y regula el régimen de derechos y obligaciones de los administradores, indicando su deber de actuar siempre en interés de la sociedad, comunicando cualquier conflicto de interés, absteniéndose del uso de información privilegiada, publicando sus retribuciones y recomendando que la mitad de los consejeros sean independientes.

6 El Informe Winter, de la Unión Europea, en 2002, incluía la obligación de las sociedades cotizadas a publicar un informe sobre buen gobierno; establecer un sistema de remuneración de consejeros y directivos; crear los comités de auditoría; promover la figura de los consejeros independientes; responsabilidad de los miembros del Consejo de Administración de probar la veracidad de la información contable, etcétera. Dicho informe recomendaba también a los Estados miembros a elaborar su propio código de buen gobierno.

7 Principios de la OCDE sobre gobierno corporativo, de 2004, que incluye la necesidad de protección de los derechos de los accionistas y de facilitar el ejercicio de sus funciones; el trato equitativo para los accionistas, incluyendo los minoritarios; la transparencia de la información corporativa relevante y las responsabilidades del Consejo de Administración. Destacar la referencia de dicho documento al papel de los grupos de interés en el gobierno corporativo. Se debe fomentar la cooperación activa entre las empresas y los grupos de interés para la creación de riqueza, empleo y la sostenibilidad de las mismas.
} 
sociedades que apelan a los mercados financieros. Su objetivo principal es conseguir una mayor independencia y transparencia en los Consejos de Administración, a los que se les asigna la función de supervisar y orientar la política de la empresa, controlar las instancias de gestión y servir de enlace con los accionistas (CNMV, 1998).

\section{Código Olivencia y RSC}

Se trata de un código que apuesta claramente por un enfoque accionarial de la empresa. Recomienda establecer como objetivo último de la misma la creación de valor para el accionista: «Recomendamos establecer como objetivo último de la compañía y, consiguientemente, como criterio que debe presidir la actuación del Consejo, la maximización del valor de la empresa o, para decirlo con una fórmula que ha arraigado en medios financieros, la creación de valor para el accionista».

No obstante, esta definición de los objetivos de la empresa queda matizada al afirmar que «el interés de los accionistas proporciona una guía de actuación que necesariamente habrá de desarrollarse respetando las exigencias impuestas por el derecho (por ejemplo, normas fiscales o medioambientales), cumpliendo de buena fe las obligaciones contractuales explícitas e implícitas concertadas con otros interesados (trabajadores, proveedores, acreedores, clientes) y, en general, observando aquellos deberes éticos que razonablemente sean apropiados para la responsable conducción de los negocios» (Lizcano, 2006).

\section{Código Aldama}

Este código se aprueba tras la publicación, en 2003, del denominado Informe Aldama
(CNMV, 2003), que contiene tres principios fundamentales (principio de transparencia, principio de lealtad y principio de libertad), así como una serie de recomendaciones en relación con la creación de comisiones delegadas y la retribución de los consejeros (Rivero, 2005).

Sin embargo, la recomendación de mayor relevancia es la que establece la necesidad de elaborar un Informe Anual de Gobierno Corporativo (IAGC) que recoja la información que debía proporcionarse a los accionistas e inversores en materia de GC. Esta recomendación se convirtió en una disposición de cumplimiento obligatorio para las sociedades cotizadas con la entrada en vigor de la denominada «Ley de Transparencia» (Ley 26/2003), que obliga a las sociedades anónimas cotizadas a elaborar un IAGC (siguiendo los principios de la Orden ECO/ 3722/2003), publicarlo en la página web de la compañía, comunicarlo a la CNMV (Comisión Nacional del Mercado de Valores) y depositarlo en el Registro Mercantil.

Se produce, de esta manera, un cambio de tendencia desde la autorregulación a la normativa legal. Ello se justificaba por la necesidad de proteger a los pequeños accionistas, de asegurar un mercado bursátil competitivo y de conseguir una mayor protección real del accionariado.

\section{Código Aldama y RSC}

El informe Aldama ofrece una visión más pluralista de las responsabilidades de la empresa, lo que supone un avance respecto al Código Olivencia. Sin embargo, dicha visión no deja de ser una exposición de motivos sin respaldo en el texto (Lizcano, 2006).

Reconoce un marco ético del GC al señalar que «la primera obligación de la empresa es $\triangleright$ 
el cumplimiento de la misión, dentro del marco legal general. No obstante, más allá del cumplimiento de las leyes y de los deberes que su misión establece, recientemente se ha venido sosteniendo que la empresa tiene otras responsabilidades sociales».

Asimismo, esta visión pluralista de la empresa queda reflejada cuando incorpora en su exposición de motivos el siguiente literal: «En el contexto de la denominada RSC en la gestión de sus negocios y en relación con sus interlocutores cada empresa podrá asumir libremente aquellas obligaciones o compromisos adicionales que desee de carácter ético o social dentro de un marco general de desarrollo sostenible, como la presentación de un triple balance económico, social y medioambiental que se discute en algunos foros, para darlos a conocer a los accionistas, empleados y a la sociedad en su conjunto, sobre la base de los principios de voluntariedad y transparencia».

Siguiendo a Lizcano, podríamos incluir este código dentro del denominado «enfoque accionarial matizado». Se hace referencia a la necesaria atención a los stakeholders pero, únicamente, como una declaración de intenciones que no tiene traslado ni desarrollo posterior en el texto.

\section{Código Conthe}

En el año 2006 se constituyó un grupo de trabajo sobre GC con el objetivo de elaborar un informe que incorporase las propuestas que se habían tenido en cuenta en las recomendaciones de la OCDE del año 2004, así como las de la propia Comisión Europea. Se pretendía, de esta manera, cubrir ciertas carencias de los códigos anteriores (CNMV, 2006). Entre sus principales recomendaciones podemos destacar las siguientes (Rivero, 2005): la publicación por las empresas de las retribuciones individualizadas de sus consejeros; la elaboración de los IAGC de acuerdo con los parámetros establecidos por el propio código; el reforzamiento de los derechos de los accionistas minoritarios; el incremento de la proporción de consejeros independientes; la promoción de las comisiones delegadas de auditoría, retribuciones y nombramientos; el aumento de las competencias de la junta de accionistas; y la promoción de la diversidad de género dentro del Consejo de Administración.

\section{Código Conthe y RSC}

Este código supuso un claro alejamiento del enfoque pluralista. Manifiesta como principal preocupación el interés del accionista y se aleja del posicionamiento de los principios de la OCDE de GC de 2004, que incorpora claramente un enfoque pluralista al gobierno de las sociedades (Lizcano, 2006).

Ello se pone de manifiesto ya en la propia introducción, al afirmar que «el código no ha entrado, de forma sustantiva, en el ámbito de la llamada RSC, que afecta principalmente a las relaciones de las compañías con otros grupos de interés distintos de sus accionistas, que no se circunscribe a las sociedades cotizadas y ha sido objeto de especial atención por una Subcomisión del Congreso de los Diputados».

De igual forma, en su recomendación 8 del capítulo II, se afirma que «el Consejo desempeñará sus funciones con unidad de propósito e independencia de la Dirección, dispensando el mismo trato a todos los accionistas, guiado por el interés de la compañía, entendido como hacer máximo, de forma sostenida, el valor económico de la empresa». 
No obstante, dicho enfoque queda matizado cuando, en esa misma recomendación, se dice que el Consejo «vele, asimismo, para que en sus relaciones con los grupos de interés, la empresa respete las leyes y los reglamentos; cumpla de buena fe sus obligaciones y contratos; respete los usos y buenas prácticas propias de los sectores y territorios donde ejerza su actividad; y observe aquellos principios adicionales de responsabilidad social que hubiera aceptado voluntariamente».

Queda igualmente matizado dicho enfoque cuando se incluye a la política de RSC como una de las políticas y estrategias generales de la sociedad que deberán ser aprobadas por el Consejo en pleno (recomendación 9).

Uno de los aspectos más llamativos de este código, en cuanto a su relación con la RSC, fue la introducción de la necesidad de promover la diversidad de género dentro del Consejo de Administración (recomendaciones 19, 20 y 21).

Código de buen gobierno de las sociedades anónimas cotizadas

Desde la aprobación del Código Conthe, en 2006, se desarrollaron diversas iniciativas legislativas que han afectado a varias de sus recomendaciones de GC. Con objeto de adaptar o suprimir aquellas recomendaciones afectadas por la nueva legislación, en junio de 2013, el Consejo de la CNMV aprobó una actualización parcial de dicho código.

Ese mismo año se creó una comisión de expertos en materia de GC con el objetivo de proponer las iniciativas y las reformas normativas necesarias para garantizar el BGC y para asesorar a la CNMV en la modificación del código anterior.

Atendiendo al primer objetivo de esta comisión, se aprobó la Ley 31/2014 para la mejora del GC de las empresas, estableciendo una serie de recomendaciones, de seguimiento voluntario, sujetas al principio de «cumplir o explicar» (Ley 31/2014).

Por otra parte, la Comisión elaboró el nuevo CBG de las sociedades anónimas cotizadas, aprobado por Acuerdo del Consejo de la CNMV, en febrero de 2015 (CNMV, 2015a). Dicho código tuvo en cuenta las recomendaciones del código anterior, los estándares internacionales de buen gobierno, las recomendaciones en materia de GC de la Comisión Europea, así como diversos documentos y propuestas de organismos internacionales y asociaciones, aportaciones doctrinales y la legislación de los países comparables.

Las características de este nuevo código siguen siendo la voluntariedad, con sujeción al principio de «cumplir» o «explicar», correspondiendo a los accionistas, inversores y, en general, los mercados, valorar las explicaciones que las sociedades cotizadas den en relación con el no seguimiento o seguimiento parcial de las recomendaciones que, en su caso, correspondan.

\section{Código de buen gobierno de las sociedades anónimas cotizadas y RSC}

Una de las principales novedades de este código, de especial relevancia para nuestro trabajo, es que incluye, por primera vez, recomendaciones específicas en materia de RSC. En él se afirma que «la importancia de la RSC es una realidad cada vez más asentada, tanto en España como en los países de nuestro entorno, que exige una adecuada atención por parte de los sistemas de gobierno corporativo de las sociedades y, por lo tanto, que no puede quedar al margen de un código de recomendaciones de buen gobierno corporativo». 
Los principios de este código recogen claramente un incremento de las exigencias para una conducta ética y responsable de las empresas, convirtiéndose la RSC en una guía de actuación de los órganos de administración de las empresas en materia de transparencia, ejemplaridad y buen gobierno.

El código contiene un total de 64 recomendaciones, tres de las cuales son recomendaciones específicas en materia de RSC $(53,54$ y 55), existiendo otras cuatro recomendaciones más $(6,12,14$ y 45) que integran la RSC en otros aspectos empresariales contemplados por el mismo.

Las recomendaciones específicas en materia de RSC del nuevo código son las siguientes:

Recomendación 53: aboga por que se incluya la política de RSC, junto con las reglas de gobierno corporativo y de los códigos internos de conducta, como materia a supervisar por alguna de las comisiones (de auditoría, de nombramientos, o una comisión específica de RSC), incluyendo un detalle de las funciones que deberá asumir tal comisión en estos ámbitos. Esta recomendación se incluye en el principio 23 del código, sobre «la composición y organización de las comisiones que, en el ejercicio de sus facultades de autoorganización, constituyan las sociedades".

Recomendación 54: establece que la política de RSC debe incluir los principios o compromisos que la empresa asuma voluntariamente en su relación con los distintos grupos de interés; las prácticas concretas en cuestiones relacionadas con accionistas, empleados, clientes, proveedores, cuestiones sociales, medio ambiente, diversidad, responsabilidad fiscal, respeto de los derechos humanos y prevención de conductas ilegales; y los mecanismos de supervisión del riesgo no financiero, la ética y la conducta empresarial, como facultad indelegable del Consejo de Administración.

Recomendación 55: establece la necesidad de información de los asuntos relacionados con la RSC en el propio informe de gestión o en un documento separado. La Directiva 2014/95 de la Unión Europea sobre divulgación de información no financiera e información sobre diversidad por parte de determinadas grandes empresas y determinados grupos supone la transposición de esta recomendación al ordenamiento jurídico.

Esta recomendación, junto con la anterior, se incluye dentro del Principio 24 del código, dedicado específicamente a la RSC, en el que se afirma que «la sociedad promoverá una política adecuada de responsabilidad social corporativa, como facultad indelegable del Consejo de Administración, ofreciendo de forma transparente información suficiente sobre su desarrollo, aplicación y resultados». Añade, además: «Por tanto, es recomendable que las empresas analicen cómo impacta su actividad en la sociedad y cómo esta impacta, a su vez, en la empresa. De esta manera, utilizando como referencia la cadena de valor, la empresa puede identificar cuestiones sociales que permitan la creación de valor compartido».

Como ya hemos comentado, además de las recomendaciones específicas sobre RSC, este código incluye cuatro recomendaciones más que integran la RSC en otros aspectos empresariales:

Recomendación 6: las sociedades cotizadas deben publicar en su página web, con antelación suficiente a la celebración de la junta general, junto con otros documentos, el informe $\square$ 
sobre la política de RSC. Esta recomendación se incluye en el Principio 6, que establece que «la junta general de accionistas debe funcionar bajo principios de transparencia y con información adecuada».

Recomendación 12: el Consejo de Administración ha de procurar conciliar el propio interés social con, según corresponda, los legítimos intereses de sus empleados, sus proveedores, sus clientes y los de los restantes grupos de interés que puedan verse afectados, así como el impacto de las actividades de la compañía en la comunidad en su conjunto y en el medio ambiente. Esta recomendación se incluye dentro del Principio 9, que establece que «el Consejo de Administración asumirá, colectiva y unitariamente, la responsabilidad directa sobre la administración social y la supervisión de la dirección de la sociedad, con el propósito común de promover el interés social».

Recomendación 14: la política de selección de consejeros ha de promover el objetivo de que, en el año 2020, el número de consejeras represente, al menos, el 30 por 100 del total de miembros del Consejo de Administración. Esta recomendación se incluye dentro del Principio 10, que establece que «el Consejo de Administración tendrá la dimensión precisa para favorecer su eficaz funcionamiento, la participación de todos los consejeros y la agilidad en la toma de decisiones, y la política de selección de consejeros promoverá la diversidad de conocimientos, experiencias y género en su composición».

Recomendación 45: la política de control y gestión de riesgos de la empresa ha de identificar los distintos tipos de riesgo, tanto financieros como no financieros (entre los que se incluyen los riesgos sociales, los riesgos medioambientales y los riesgos por reputación) a los que se enfrenta. Esta recomendación se incluye en el Principio 21, que establece que «la sociedad dispondrá de una función de control y gestión de riesgos ejercida por una unidad o departamento interno, bajo la supervisión directa de la Comisión de Auditoría o, en su caso, de otra comisión especializada del Consejo de Administración».

El literal de estas recomendaciones, tal y como se explicitan en el mencionado código, se recoge en el Anexo I de este trabajo.

\section{Objetivo del trabajo}

El objetivo de este trabajo es analizar el nivel de cumplimiento de las recomendaciones sobre RSC contenidas en el nuevo CBG de las sociedades anónimas cotizadas por parte de las empresas del IBEX. En la Tabla 1 se relacionan las empresas pertenecientes al IBEX35 , a la fecha de elaboración de este estudio, indicando el sector y subsector al que pertenecen, así como su pertenencia o no al índice Dow Jones Sustainability (DJS). La pertenencia a dicho índice presupone el cumplimiento por parte de las empresas de los criterios de buenas prácticas en RSC (trabajan por la sostenibilidad medioambiental, el desarrollo de relaciones positivas con los grupos de interés y el apoyo y preservación de los derechos humanos universales).

\section{Metodología}

Para el cumplimiento de dicho objetivo se ha revisado la información que dichas empresas han declarado en su IAGC y presentado $D$ 
TABLA 1

EMPRESAS DEL IBEX-35

\begin{tabular}{|c|c|c|c|}
\hline Empresa & Sector & Subsector & \\
\hline Abertis Infraestructuras, S.A. & S3 & Autopistas y aparcamientos & (DJS) \\
\hline Acciona,S.A. & $\mathrm{S} 2$ & Construcción & (DJS) \\
\hline Acerinox, S.A. & $\mathrm{S} 2$ & Mineral, metales y transformación & \\
\hline ACS, Actividades de construcción y servicios, S.A. & S2 & Construcción & \\
\hline Aena, S.A. & S3 & Transporte y distribución & \\
\hline Amadeus It Group, S.A. & S6 & Electrónica y software & (DJS) \\
\hline Arcelormittal, S.A. & S2 & Mineral, metales y transformación & \\
\hline Banco Bilbao Vizcaya Argentaria, S.A. & S1 & Bancos y cajas de ahorro & \\
\hline Banco de Sabadell, S.A. & S1 & Bancos y cajas de ahorro & \\
\hline Banco Santander, S.A. & S1 & Bancos y cajas de ahorro & (DJS) \\
\hline Bankia, S.A. & S1 & Bancos y cajas de ahorro & (DJS) \\
\hline Bankinter,S.A. & S1 & Bancos y cajas de ahorro & \\
\hline Caixabank, S.A. & S1 & Bancos y cajas de ahorro & (DJS) \\
\hline Cellnex Telecom, S.A. & S6 & Telecomunicaciones y otros & \\
\hline DIA, Distribuidora Internacional de Alimentación, S.A. & S3 & Comercio & \\
\hline Enagas, S.A. & S4 & Electricidad y gas & (DJS) \\
\hline Endesa, S.A. & S4 & Electricidad y gas & (DJS) \\
\hline Ferrovial, S.A. & $\mathrm{S} 2$ & Construcción & (DJS) \\
\hline Gas Natural SDG, S.A. & S4 & Electricidad y gas & (DJS) \\
\hline Grifols, S.A. & S5 & Productos farmaceuticos y biotecnología & \\
\hline Iberdrola, S.A. & S4 & Electricidad y gas & (DJS) \\
\hline Indra Sistemas, S.A., Serie A & S6 & Electrónica y software & (DJS) \\
\hline INDITEX, Industria de Diseño Textil, S.A. & S5 & Textil, vestido y calzado & (DJS) \\
\hline Inmobiliaria Colonial, S.A. & $\mathrm{S} 1$ & Inmobiliarias y otros & \\
\hline International Consolidat. Airlines Group & S3 & Transporte y distribución & \\
\hline Mapfre, S.A. & S1 & Seguros & \\
\hline Mediaset España Comunicacion, S.A. & S3 & Medios de comunicación y publicidad & \\
\hline Melia Hotels International, S.A. & S3 & Ocio, turismo y hostelería & \\
\hline Merlin Properties, SOCIMI, S.A. & S1 & SOCIMI & \\
\hline Red Electrica Corporacion, S.A. & S4 & Electricidad y gas & (DJS) \\
\hline Repsol, S.A. & S4 & Petróleo & (DJS) \\
\hline Siemens Gamesa Renewable Energy, S.A. & S2 & Fabricación y montaje bienes de equipo & (DJS) \\
\hline Técnicas Reunidas, S.A. & $\mathrm{S} 2$ & Ingeniería y otro & \\
\hline Telefónica, S.A. & S6 & Telecomunicaciones y otros & (DJS) \\
\hline Viscofan, S.A. & S5 & Alimentación y bebidas & \\
\hline
\end{tabular}

ante la CNMV, correspondientes a los ejercicios 2015 y 2016, años de vigencia de dicho código. Hemos definido 7 variables, que se corresponden con las recomendaciones que se incluyen en el código, relacionadas con la RSC, y que son las siguientes.
R6: publicación en web de informes para la junta de accionistas.

R12: interés social y medioambiental.

R14: política de selección de consejeros.

R45: contenido de la política de control y gestión de riesgos. 
R53: supervisión cumplimiento reglas GC, códigos internos de conducta y RSC.

R54: contenido mínimo política de RSC.

R55: información sobre RSC en informe de gestión o informe especial.

Se ha revisado el grado de cumplimiento de las recomendaciones mencionadas, atendiendo a los criterios de «cumple», «cumple parcialmente» o «explica», de cada una de las empresas en los dos ejercicios mencionados.

En el Anexo II se recogen los resultados individualizados para cada empresa.

Por último, se ha analizado el nivel de cumplimiento por sectores de actividad, así como el de aquellas empresas del IBEX incluidas, a su vez, en el índice DJS.

\section{Resultados}

En el año 2015, primer año de vigencia de este código, el porcentaje de cumplimiento total de las recomendaciones de RSC contenidas en el mismo alcanzaba el 87,3 por 100.

Si nos circunscribimos a las 17 empresas del IBEX que se incluyen en el índice DJS, este porcentaje de cumplimiento, como se podía esperar, es aún mayor (92,4 por 100).

Por lo tanto, a pesar de ser la primera vez en nuestro país que un CBGC incluye recomendaciones específicas en materia de RSC (excluidas expresamente en el código anterior), el nivel de cumplimiento de las mismas durante el primer año de vigencia es similar al del resto de recomendaciones, muchas de las cuales ya venían incorporándose en códigos anteriores ${ }^{8}$.

Ya en 2015 existían cuatro sectores de actividad (servicios financieros e inmobiliarios; petróleo y energía; bienes de consumo; y tecnología y telecomunicaciones) con niveles de $\triangleright$

8 Según el último informe agrupado elaborado por la CNMV sobre el gobierno corporativo de las entidades emisoras de valores admitidos a negociación en mercados secundarios oficiales, correspondiente al ejercicio 2015 (CNMV 2015), en promedio, las sociedades del IBEX-35 cumplían el 88,4 por 100 del Código de buen gobierno.

TABLA 2

PORCENTAJE DE CUMPLIMIENTO DE RECOMENDACIONES RSC EN EMPRESAS DEL IBEX POR SECTORES

\begin{tabular}{|c|c|c|c|c|c|c|c|c|c|c|c|c|c|c|}
\hline \multirow{3}{*}{ Sector } & \multicolumn{2}{|c|}{ R6 } & \multicolumn{2}{|c|}{ R12 } & \multicolumn{2}{|c|}{ R14 } & \multicolumn{2}{|c|}{ R45 } & \multicolumn{2}{|c|}{ R53 } & \multicolumn{2}{|c|}{ R54 } & \multicolumn{2}{|c|}{ R55 } \\
\hline & \multicolumn{2}{|c|}{$\begin{array}{l}\text { Publicación en } \\
\text { web de informes } \\
\text { para la junta }\end{array}$} & \multicolumn{2}{|c|}{ Interés social } & \multicolumn{2}{|c|}{$\begin{array}{l}\text { Política de } \\
\text { selección }\end{array}$} & \multicolumn{2}{|c|}{$\begin{array}{l}\text { Contenido de } \\
\text { la política de } \\
\text { control y gestión } \\
\text { de riesgos }\end{array}$} & \multicolumn{2}{|c|}{$\begin{array}{l}\text { Supervisión } \\
\text { cumplimiento } \\
\text { reglas gob. corp., } \\
\text { códigos internos con- } \\
\text { ducta y política RSC }\end{array}$} & \multicolumn{2}{|c|}{$\begin{array}{c}\text { Contenido } \\
\text { mínimo de la } \\
\text { política RSC }\end{array}$} & \multicolumn{2}{|c|}{$\begin{array}{l}\text { Información } \\
\text { sobre RSC en } \\
\text { informe gestión } \\
\text { o informe } \\
\text { especial }\end{array}$} \\
\hline & 2015 & 2016 & 2015 & 2016 & 2015 & 2016 & 2015 & 2016 & 2015 & 2016 & 2015 & 2016 & 2015 & 2016 \\
\hline S1 & 66 & 100 & 100 & 100 & 89 & 100 & 100 & 100 & 78 & 78 & 100 & 100 & 100 & 100 \\
\hline $\mathrm{S} 2$ & 43 & 57 & 100 & 100 & 57 & 71 & 100 & 89 & 57 & 71 & 86 & 86 & 100 & 100 \\
\hline S3 & 83 & 83 & 100 & 100 & 66 & 66 & 100 & 100 & 66 & 83 & 66 & 83 & 100 & 100 \\
\hline S4 & 100 & 100 & 100 & 100 & 50 & 66 & 100 & 100 & 100 & 100 & 100 & 100 & 100 & 100 \\
\hline S5 & 66 & 100 & 100 & 100 & 100 & 100 & 100 & 100 & 66 & 66 & 100 & 100 & 100 & 100 \\
\hline S6 & 100 & 75 & 100 & 100 & 75 & 100 & 100 & 100 & 100 & 100 & 75 & 100 & 100 & 100 \\
\hline Total & 74,3 & 85,7 & 100 & 100 & 71,4 & 82,8 & 100 & 97,1 & 77,1 & 82,8 & 88,6 & 94,3 & 100 & 100 \\
\hline Empresas & $26 / 35$ & $30 / 35$ & $35 / 35$ & $35 / 35$ & $25 / 35$ & $29 / 35$ & $35 / 35$ & $34 / 35$ & $27 / 35$ & $29 / 35$ & $31 / 35$ & $33 / 35$ & $35 / 35$ & $35 / 35$ \\
\hline (DJS) & 88,2 & 94,1 & 100 & 100 & 76,5 & 82,3 & 94,1 & 94,1 & 94,1 & 94,1 & 94,1 & 94,1 & 100 & 100 \\
\hline Empresas & $15 / 17$ & $16 / 17$ & $17 / 17$ & $17 / 17$ & $13 / 17$ & $14 / 17$ & $16 / 17$ & $16 / 17$ & $16 / 17$ & $16 / 17$ & $16 / 17$ & $16 / 17$ & $17 / 17$ & $17 / 17$ \\
\hline
\end{tabular}


cumplimiento medio de las recomendaciones sobre RSC superiores al 90 por 100.

Las recomendaciones específicas de RSC (R53, R54 y R55) presentaban, en 2015, un nivel de cumplimiento del 88,6 por 100 de promedio, mientras que las recomendaciones que integran la RSC en otros aspectos empresariales contemplados en el código ( $\mathrm{R} 6, \mathrm{R} 12$, R14 y R45) lo hacían en un 86,4 por 100 . Tres recomendaciones (R12, R45 y R55) presentaban un nivel de cumplimiento del 100 por 100 , siendo la R14 y la R6 las recomendaciones con menor nivel de cumplimiento.

En el año 2016 se producen importantes avances. El porcentaje de cumplimiento total de dichas recomendaciones se sitúa en el 91,8 por 100 (4,5 puntos más que en 2015) y el de las empresas del IBEX incluidas en el índice DJS, en el 94,1 por 100 (1,7 puntos más que en 2015).

Este año el 62,9 por 100 de las empresas del IBEX cumplen con el 100 por 100 de las recomendaciones sobre RSC del mencionado código; en el caso de las empresas del IBEX incluidas en el índice DJS este porcentaje se eleva hasta el 70,6 por 100 . Tan sólo cuatro empresas del IBEX mantienen porcentajes de cumplimiento total por debajo del 85 por $100^{9}$.

Cuatro sectores de actividad (servicios financieros e inmobiliarios; petróleo y energía; bienes de consumo; y tecnología y telecomunicaciones) mantienen niveles globales de

9 ACS, Ferrovial, Melia Hotels y Técnicas Reunidas cumplimiento cercanos al 100 por 100 en este ejercicio.

Se producen importantes avances en el porcentaje de cumplimiento de los restantes sectores, incluido el de materiales básicos, industria y construcción, que presentaba el menor porcentaje de cumplimiento en 2015.

Durante el año 2016 se produce un importante avance en el nivel de cumplimiento de todas las recomendaciones de RSC en las empresas del IBEX, pero fundamentalmente en aquellas que presentaban un menor nivel de cumplimiento en el año anterior (la R14, que pasa del 71,4 por 100 de cumplimiento en 2015 al 82,8 por 100 en el 2016; y la R6, que pasa del 74,3 por 100 de nivel de cumplimiento en 2015 al 85,7 por 100 en el año 2016).

Las recomendaciones específicas de RSC (R53, R54 y R55) alcanzan ya un nivel de cumplimiento en 2016 del 92,4 por 100 , mientras que las recomendaciones que integran la RSC en otros aspectos empresariales (R6, R12, R14 y R45) se sitúan ya en el 91,4 por 100 .

Esta realidad, sin embargo, difiere de la presentada por la CNMV en su informe de GC de las entidades emisoras de valores admitidos a negociación en mercados secundarios oficiales del ejercicio 2015 (último publicado) (CNMV, 2015b).

De este informe se extrae que los niveles medios de cumplimiento de las recomendaciones de RSC de la totalidad de empresas cotizadas de nuestro país en 2015 están muy por debajo de los presentados para las empresas del IBEX. $\square$

TABLA 3

PORCENTAJE DE CUMPLIMIENTO DE RECOMENDACIONES SOBRE RSC EN LAS EMPRESAS DEL IBEX EN RELACIÓN AL TOTAL DE EMPRESAS COTIZADAS

\begin{tabular}{|l|c|c|c|c|c|c|c|}
\hline \multicolumn{1}{|c|}{ Recomendaciones } & R6 & R12 & $\mathbf{R 1 4}$ & $\mathbf{R 4 5}$ & $\mathbf{R 5 3}$ & $\mathbf{R 5 4}$ & R55 \\
\hline Porcentaje cumplimiento empresas IBEX & 74,3 & 100 & 71,4 & 100 & 77,1 & 88,6 & 100 \\
Porcentaje cumplimiento total empresas cotizadas & 39,4 & 99,3 & 52,6 & 91,2 & 67,2 & 64,9 & 64,2 \\
Diferencia & 34,9 & 0,7 & 18,8 & 8,8 & 9,9 & 23,7 & 35,8 \\
\hline
\end{tabular}

Fuente: elaboración propia. 


\section{Conclusiones}

Los resultados de este trabajo ponen de manifiesto la rapidez con que las empresas del IBEX han incorporado en sus políticas de GC las recomendaciones de RSC contenidas en el Código de buen gobierno de las sociedades cotizadas de 2015.

Además, la evolución, claramente favorable, de dicho nivel de cumplimiento, en los dos primeros años de vigencia del mencionado código, evidencia que cada vez son más las empresas dispuestas a asumir tales recomendaciones y los cambios que las mismas suponen en el papel tradicional de los Consejos de Administración y de los propios administradores.

Podríamos concluir, por lo tanto, que este nuevo código está colaborando en la asunción de la RSC como un componente fundamental del GC, favoreciéndose, de este modo, el tránsito de un modelo orientado al accionista hacia un modelo orientado a los stakeholders, al menos en las empresas de mayor capitalización bursátil de nuestro país. Sin embargo, el análisis de los niveles de cumplimiento de estas recomendaciones en las empresas del IBEX, en comparación con los niveles de cumplimiento de la totalidad de empresas cotizadas de nuestro país, nos puede llevar a concluir que en la medida en que las empresas están dispuestas a asumir los principios de la RSC en sus GC mantiene una relación directa con su nivel de capitalización.

\section{Bibliografía}

[1] ALEJOS, C. L. (2015). «Gobierno corporativo ¿y? responsable». Cuadernos de la Cátedra «la Caixa» de Responsabilidad Social de La Empresa y Gobierno Corporativo, n. .28 .

[2] CADBURY, A.S. (1992). Informe de comité sobre los aspectos financieros del gobierno corporativo. (Recuperado el 5 de septiembre de 2017). Disponible en: https://scholar.google.es/scholar?hl=es\&q=Informe+Cadbury+$d e+1992+\& b t n G=\& / r=$

[3] CNMV (1998). El Gobierno de las Sociedades Cotizadas (Código Olivencia). (Recuperado el 1 de septiembre de 2017). Disponible en: https://www.cnmv.es/DocPortal/Publicaciones/ CodigoGov/govsocot.pdf

[4] CNMV (2003). Informe de la Comisión Especial para el Fomento de la Transparencia y Seguridad en los Mercados y en las Sociedades Cotizadas (Informe Aldama). (Recuperado el 3 de septiembre de 2017). Disponible en: https://www.cnmv.es/DocPortal/Publicaciones/ CodigoGov/INFORMEFINAL.PDF

[5] CNMV (2006). Informe del Grupo Especial de Trabajo sobre el Buen gobierno de las Sociedades Cotizadas (Código Conthe). (Recuperado el 28 de agosto de 2017). Disponible en: https://www.cnmv.es/Portal/ / verDoc. $a x d ? t=\% 7 B 98 a 0 b 978-5644-4 d c 1$ a447-2b33ac5f34bc\%7D

[6] CNMV (2015a). Código de buen gobierno de las sociedades cotizadas. (Recuperado el 29 de agosto de 2017). Disponible en: $h$ ttp:// www.cnmv.es/DocPortal/Publicaciones/ CodigoGov/Codigo_buen_gobierno.pdf

[7] CNMV (2015b). Informe de Gobierno Corporativo de las entidades emisoras de valores admitidos a negociación en mercados secundarios oficiales. (Recuperado el 20 de agosto de 2017). Disponible en: https://www. cnmv.es/DocPortal/Publicaciones/Informes/ IAGC_2015.pdf

[8] COMISION EUROPEA (2001). Resumen de las observaciones y recomendaciones del grupo de alto nivel de expertos en Derecho de sociedades (Informe Winter). (Recuperado el 7 de septiembre de 2017). Disponible en: http://www.icjce.es/images/pdfs/TECNICA/ B1-ComisiónEuropea/B.1.21-CE-DSyGCModernizlegis/InformeWinter(TradICAC)04112002.PDF

[9] COMMITTEE OF SPONSORING ORGANIZATIONS OF THE TREADWAY COMMISSION (1987). Informe COSO. (Recuperado el 5 de septiembre de 2017). Disponible en: http://www.contabilidad.com.py/articulos_ 75_informe-coso-historia.html 
[10] FERRUZ, L.; MARCO, I. y ACERO, I. (2010). «Códigos de Buen gobierno: Un análisis comparativo. Especial incidencia en el caso español». Revista de Ciencias Sociales, n. 46.

[11] FUSHIMI, J. (2011). «Crisis financiera global y corporate governance». Oikonomos, año 2 , vol. 1, pp. 13-53.

[12] HAMPEL, L. (1998). Informe Hampel.Comité sobre el gobierno corporativo. (Recuperado el 8 de septiembre de 2017). Disponible en: http:/www. limalaw.blogspot.com.es/2012/05/ gobierno-corporativo-en-inglaterra-el_244. html

[13] JAMALI, D.; SAFIEDDINE, A. M. y RABBATH, M. (2008). «Corporate Governance and Corporate Social Responsibility Synergies and Interrelationships». Corporate Governance: An International Review, vol. 5, n.․ 16, pp. 443-459.

[14] LEY 26/2003 (2003). Ley por la que se modifican la Ley 24/1988, de 28 de julio, del Mercado de Valores, y el texto refundido de la Ley de Sociedades Anónimas, aprobado por el Real Decreto Legislativo 1564/1989, de 22 de diciembre, con el fin de reforzar la transparencia de las sociedades anónimas cotizadas. (Recuperado el 5 de septiembre de 2017).
Disponible en: http://noticias.juridicas.com/ base_datos/Privado//26-2003.html

[15] LEY 31/2014, (2014). Ley por la que se modifica la Ley de Sociedades de Capital. (Recuperado el 8 de septiembre de 2017). Disponible en: https://es. search.yahoo.com/search?fr=mcafee\&type $=C 111 E S O D 20170410 \& p=L e y+31 \%$ $2 F 2014 \% 2 C+d e+3+d e+d i c i e m b r e$

[16] LIZCANO, J. L. (2006). «Buen gobierno y responsabilidad social corporativa». Partida Doble, n.․ 182, pp. 20-35.

[17] LIZCANO, J.L. y MONEVA, J. (2004). «Marco conceptual de la responsabilidad social corporativa». AECA.

[18] OCDE (2004). Principios de Gobierno Corporativo de la OCDE. (Recuperado el 7 de septiembre de 2017). Disponible en: http:// www.oecd.org/daf/ca/corporategovernanceprinciples/37191543.pdf

[19] RIVERO, P. (2005). «Responsabilidad Social y Gobierno Corporativo: Información y Transparencia». Revista asturiana de economía, n. ${ }^{\circ} 34$.

[20] VIENOT, M. (1995). Le Conseil D'administración des Societes Cotees. Rapport Vienot. (Recuperado el 10 de septiembre de 2017). Disponible en: $h$ ttps://doi.org/10.07.95 


\section{ANEXO I \\ RECOMENDACIONES DE RSC INCLUIDAS EN EL CÓDIGO DE BUEN GOBIERNO DE LAS SOCIEDADES ANÓNIMAS COTIZADAS DE 2015}

\section{Recomendación 6:}

Que las sociedades cotizadas que elaboren los informes que se citan a continuación, ya sea de forma preceptiva o voluntaria, los publiquen en su página web con antelación suficiente a la celebración de la junta general ordinaria, aunque su difusión no sea obligatoria:

a) Informe sobre la independencia del auditor.

b) Informes de funcionamiento de las comisiones de auditoría y de nombramientos y retribuciones.

c) Informe de la comisión de auditoría sobre operaciones vinculadas.

d) Informe sobre la política de responsabilidad social corporativa.

\section{Recomendación 12:}

Que el consejo de administración desempeñe sus funciones con unidad de propósito e independencia de criterio, dispense el mismo trato a todos los accionistas que se hallen en la misma posición y se guíe por el interés social, entendido como la consecución de un negocio rentable y sostenible a largo plazo, que promueva su continuidad y la maximización del valor económico de la empresa.

Y que en la búsqueda del interés social, además del respeto de las leyes y reglamentos y de un comportamiento basado en la buena fe, la ética y el respeto a los usos y a las buenas prácticas comúnmente aceptadas, procure conciliar el propio interés social con, según corresponda, los legítimos intereses de sus empleados, sus proveedores, sus clientes y los de los restantes grupos de interés que puedan verse afectados, así como el impacto de las actividades de la compañía en la comunidad en su conjunto y en el medio ambiente.

\section{Recomendación 14:}

Que el consejo de administración apruebe una política de selección de consejeros que:

a) Sea concreta y verificable.

b) Asegure que las propuestas de nombramiento o reelección se fundamenten en un análisis previo de las necesidades del consejo de administración.

c) Favorezca la diversidad de conocimientos, experiencias y género.

Que el resultado del análisis previo de las necesidades del consejo de administración se recoja en el informe justificativo de la comisión de nombramientos que se publique al convocar la junta general de accionistas a la que se someta la ratificación, el nombramiento o la reelección de cada consejero.

Y que la política de selección de consejeros promueva el objetivo de que en el año 2020 el número de consejeras represente, al menos, el 30 por 100 del total de miembros del consejo de administración.

La comisión de nombramientos verificará anualmente el cumplimiento de la política de selección de consejeros y se informará de ello en el informe anual de gobierno corporativo.

\section{Recomendación 45:}

Que la política de control y gestión de riesgos identifique al menos:

a) Los distintos tipos de riesgo, financieros y no financieros (entre otros los operativos, tecnológicos, legales, sociales, medio ambientales, políticos y reputacionales) a los que se enfrenta la sociedad, incluyendo entre los financieros o económicos, los pasivos contingentes y otros riesgos fuera de balance.

b) La fijación del nivel de riesgo que la sociedad considere aceptable.

c) Las medidas previstas para mitigar el impacto de los riesgos identificados, en caso de que llegaran a materializarse. 
d) Los sistemas de información y control interno que se utilizarán para controlar y gestionar los citados riesgos, incluidos los pasivos contingentes o riesgos fuera de balance.

\section{Recomendación 53:}

Que la supervisión del cumplimiento de las reglas de gobierno corporativo, de los códigos internos de conducta y de la política de responsabilidad social corporativa se atribuya a una o se reparta entre varias comisiones del consejo de administración que podrán ser la comisión de auditoría, la de nombramientos, la comisión de responsabilidad social corporativa, en caso de existir, o una comisión especializada que el consejo de administración, en ejercicio de sus facultades de autoorganización, decida crear al efecto, a las que específicamente se les atribuyan las siguientes funciones mínimas:

a) La supervisión del cumplimiento de los códigos internos de conducta y de las reglas de gobierno corporativo de la sociedad.

b) La supervisión de la estrategia de comunicación y relación con accionistas e inversores, incluyendo los pequeños y medianos accionistas.

c) La evaluación periódica de la adecuación del sistema de gobierno corporativo de la sociedad, con el fin de que cumpla su misión de promover el interés social y tenga en cuenta, según corresponda, los legítimos intereses de los restantes grupos de interés.

d) La revisión de la política de responsabilidad corporativa de la sociedad, velando por que esté orientada a la creación de valor.

e) El seguimiento de la estrategia y prácticas de responsabilidad social corporativa y la evaluación de su grado de cumplimiento.

f) La supervisión y evaluación de los procesos de relación con los distintos grupos de interés.

g) La evaluación de todo lo relativo a los riesgos no financieros de la empresa -incluyendo los operativos, tecnológicos, legales, sociales, medio ambientales, políticos y reputacionales.

h) La coordinación del proceso de reporte de la información no financiera y sobre diversidad, conforme a la normativa aplicable y a los estándares internacionales de referencia.

\section{Recomendación 54:}

Que la política de responsabilidad social corporativa incluya los principios o compromisos que la empresa asuma voluntariamente en su relación con los distintos grupos de interés e identifique al menos:

a) Los objetivos de la política de responsabilidad social corporativa y el desarrollo de instrumentos de apoyo.

b) La estrategia corporativa relacionada con la sostenibilidad, el medio ambiente y las cuestiones sociales.

c) Las prácticas concretas en cuestiones relacionadas con: accionistas, empleados, clientes, proveedores, cuestiones sociales, medio ambiente, diversidad, responsabilidad fiscal, respeto de los derechos humanos y prevención de conductas ilegales.

d) Los métodos o sistemas de seguimiento de los resultados de la aplicación de las prácticas concretas señaladas en la letra anterior, los riesgos asociados y su gestión.

e) Los mecanismos de supervisión del riesgo no financiero, la ética y la conducta empresarial.

f) Los canales de comunicación, participación y diálogo con los grupos de interés.

g) Las prácticas de comunicación responsable que eviten la manipulación informativa y protejan la integridad y el honor.

\section{Recomendación 55:}

Que la sociedad informe, en un documento separado o en el informe de gestión, sobre los asuntos relacionados con la responsabilidad social corporativa, utilizando para ello alguna de las metodologías aceptadas internacionalmente. 


\section{ANEXO II}

CUMPLIMIENTO DE LAS RECOMENDACIONES SOBRE RSC CONTENIDAS EN EL NUEVO CÓDIGO DE BUEN GOBIERNO DE LAS SOCIEDADES ANÓNIMAS COTIZADAS DE 2015, POR PARTE DE LAS EMPRESAS DEL IBEX-35

\begin{tabular}{|c|c|c|c|c|c|c|c|c|c|c|c|c|c|c|c|c|c|c|c|c|c|c|c|}
\hline \multirow{2}{*}{ Empresa } & \multirow{2}{*}{ Año } & \multicolumn{3}{|c|}{ R6 } & \multicolumn{3}{|c|}{ R12 } & \multicolumn{3}{|c|}{ R14 } & \multicolumn{3}{|c|}{ R45 } & \multicolumn{3}{|c|}{ R53 } & \multicolumn{3}{|c|}{ R54 } & \multicolumn{3}{|c|}{ R55 } & \multirow{2}{*}{$\begin{array}{c}\% \\
\text { Cumpli. }\end{array}$} \\
\hline & & C & CP & EX & C & CP & EX & C & CP & EX & C & CP & EX & C & CP & EX & C & CP & EX & C & CP & EX & \\
\hline \multirow{2}{*}{ Abertis Infraestructuras, S.A. } & 2015 & * & & & * & & & * & & & * & & & * & & & * & & & * & & & 100 \\
\hline & 2016 & * & & & * & & & * & & & * & & & * & & & * & & & * & & & 100 \\
\hline & 2015 & & * & & * & & & & * & & * & & & * & & & * & & & * & & & 71 \\
\hline Acciona, S.A. & 2016 & * & & & * & & & & * & & * & & & * & & & * & & & * & & & 85 \\
\hline & 2015 & * & & & * & & & * & & & * & & & * & & & * & & & * & & & 100 \\
\hline Acerinox, S.A. & 2016 & * & & & * & & & * & & & * & & & * & & & * & & & * & & & 100 \\
\hline ACS, Actividades de Const. & 2015 & & * & & * & & & & * & & * & & & & * & & * & & & * & & & 57 \\
\hline y Servicios, S.A. & 2016 & & * & & * & & & & * & & * & & & * & & & * & & & * & & & 71 \\
\hline & 2015 & * & & & * & & & * & & & * & & & & * & & * & & & * & & & 85 \\
\hline Aena, S.A. & 2016 & * & & & * & & & * & & & * & & & * & & & * & & & * & & & 100 \\
\hline & 2015 & * & & & * & & & * & & & * & & & * & & & * & & & * & & & 100 \\
\hline Amadeus It Group, S.A. & 2016 & * & & & * & & & * & & & * & & & * & & & * & & & * & & & 100 \\
\hline A roolormittol $c \wedge$ & 2015 & * & & & * & & & * & & & * & & & * & & & * & & & * & & & 100 \\
\hline Arcelormıttal, S.A. & 2016 & * & & & * & & & * & & & * & & & * & & & * & & & * & & & 100 \\
\hline Banco Bilbao Vizcaya & 2015 & * & & & * & & & * & & & * & & & * & & & * & & & * & & & 100 \\
\hline Argentaria, S.A. & 2016 & * & & & * & & & * & & & * & & & * & & & * & & & * & & & 100 \\
\hline & 2015 & & * & & * & & & * & & & * & & & * & & & * & & & * & & & 85 \\
\hline Banco de Sabadell, S.A. & 2016 & * & & & * & & & * & & & * & & & * & & & * & & & * & & & 100 \\
\hline & 2015 & * & & & * & & & * & & & * & & & * & & & * & & & * & & & 100 \\
\hline Banco Santander, S.A. & 2016 & * & & & * & & & * & & & * & & & * & & & * & & & * & & & 100 \\
\hline & 2015 & * & & & * & & & * & & & * & & & * & & & * & & & * & & & 100 \\
\hline Bankia, S.A. & 2016 & * & & & * & & & * & & & * & & & * & & & * & & & * & & & 100 \\
\hline & 2015 & * & & & * & & & * & & & * & & & * & & & * & & & * & & & 100 \\
\hline Bankinter, S.A. & 2016 & * & & & * & & & * & & & * & & & * & & & * & & & * & & & 100 \\
\hline Emnreca & Ãn & & R6 & & & R12 & & & R14 & & & R45 & & & R53 & & & R54 & & & R55 & & $\%$ \\
\hline Empresa & Ano & C & CP & EX & C & CP & EX & C & CP & EX & C & CP & EX & C & CP & EX & C & CP & EX & C & CP & EX & Cumpli. \\
\hline & 2015 & * & & & * & & & * & & & * & & & * & & & * & & & * & & & 100 \\
\hline Calxabank, S.A. & 2016 & * & & & * & & & * & & & * & & & * & & & * & & & * & & & 100 \\
\hline & 2015 & * & & & * & & & & * & & * & & & * & & & 然 & * & & * & & & 71 \\
\hline Cellnex Telecom, S.A. & 2016 & & * & & * & & & * & & & * & & & * & & & * & & & * & & & 85 \\
\hline DIA. Distribuidora Internacional & 2015 & * & & & * & & & * & & & * & & & * & & & * & & & * & & & 100 \\
\hline de Alimentación S.A. & 2016 & * & & & * & & & * & & & * & & & * & & & * & & & * & & & 100 \\
\hline Enagas SA & 2015 & * & & & * & & & & * & & * & & & * & & & * & & & * & & & 85 \\
\hline Enagas, S.A. & 2016 & * & & & * & & & * & & & * & & & * & & & * & & & * & & & 100 \\
\hline Fndece $\mathrm{SA}$ & 2015 & * & & & * & & & * & & & * & & & * & & & * & & & * & & & 100 \\
\hline Endesa, S.A. & 2016 & * & & & * & & & * & & & * & & & * & & & * & & & * & & & 100 \\
\hline & 2015 & & * & & * & & & * & & & * & & & & * & & & * & & * & & & 57 \\
\hline Ferrovial, S.A. & 2016 & & * & & * & & & * & & & * & & & & * & & & * & & * & & & 57 \\
\hline & 2015 & * & & & * & & & & * & & * & & & * & & & * & & & * & & & 85 \\
\hline Gas Natural SDG, S.A. & 2016 & * & & & * & & & & * & & * & & & * & & & * & & & * & & & 85 \\
\hline & 2015 & & & * & * & & & * & & & * & & & * & & & * & & & * & & & 85 \\
\hline Grifols, S.A. & 2016 & * & & & * & & & * & & & * & & & * & & & * & & & * & & & 100 \\
\hline Iherdrola SA & 2015 & * & & & * & & & * & & & * & & & * & & & * & & & * & & & 100 \\
\hline Iberdrola, S.A. & 2016 & * & & & * & & & * & & & * & & & * & & & * & & & * & & & 100 \\
\hline Indra Sistemas, S.A., & 2015 & * & & & * & & & * & & & * & & & * & & & * & & & * & & & 100 \\
\hline Serie A & 2016 & * & & & * & & & * & & & * & & & * & & & * & & & * & & & 100 \\
\hline INDITEX. Industria de & 2015 & * & & & * & & & * & & & * & & & * & & & * & & & * & & & 100 \\
\hline Diseño Textil, S.A. & 2016 & * & & & * & & & * & & & * & & & * & & & * & & & * & & & 100 \\
\hline & 2015 & & * & & * & & & & * & & * & & & * & & & * & & & * & & & 71 \\
\hline Inmobiliaria Colonial, S.A. & 2016 & * & & & * & & & * & & & * & & & * & & & * & & & * & & & 100 \\
\hline
\end{tabular}




\begin{tabular}{|c|c|c|c|c|c|c|c|c|c|c|c|c|c|c|c|c|c|c|c|c|c|c|c|}
\hline \multirow{2}{*}{ Empresa } & \multirow{2}{*}{ AÑO } & \multicolumn{3}{|c|}{ R6 } & \multicolumn{3}{|c|}{ R12 } & \multicolumn{3}{|c|}{ R14 } & \multicolumn{3}{|c|}{ R45 } & \multicolumn{3}{|c|}{ R53 } & \multicolumn{3}{|c|}{ R54 } & \multicolumn{3}{|c|}{ R55 } & \multirow{2}{*}{$\begin{array}{c}\% \\
\text { Cumpli. }\end{array}$} \\
\hline & & C & CP & EX & C & CP & EX & C & CP & EX & C & CP & EX & C & CP & EX & C & CP & EX & C & CP & EX & \\
\hline \multirow{2}{*}{$\begin{array}{l}\text { International Consolidat. } \\
\text { Airlines Group }\end{array}$} & 2015 & * & & & * & & & * & & & * & & & * & & & * & & & * & & & 100 \\
\hline & 2016 & * & & & * & & & * & & & * & & & * & & & * & & & * & & & 100 \\
\hline \multirow{2}{*}{ Mapfre, S.A. } & 2015 & & * & & * & & & * & & & * & & & & * & & * & & & * & & & 71 \\
\hline & 2016 & * & & & * & & & * & & & * & & & & * & & * & & & * & & & 85 \\
\hline \multirow{2}{*}{$\begin{array}{l}\text { Mediaset España } \\
\text { Comunicación, S.A. }\end{array}$} & 2015 & * & & & * & & & & * & & * & & & * & & & & * & & * & & & 71 \\
\hline & 2016 & * & & & * & & & & * & & * & & & * & & & * & & & * & & & 85 \\
\hline \multirow{2}{*}{$\begin{array}{l}\text { Melia Hotels International, } \\
\text { S.A. }\end{array}$} & 2015 & & * & & * & & & & & * & * & & & & * & & & * & & * & & & 43 \\
\hline & 2016 & & * & & * & & & & & * & * & & & & * & & & * & & * & & & 43 \\
\hline \multirow{2}{*}{$\begin{array}{l}\text { Merlin Properties, SOCIMI, } \\
\text { S.A. }\end{array}$} & 2015 & * & & & * & & & * & & & * & & & & * & & * & & & * & & & 85 \\
\hline & 2016 & * & & & * & & & * & & & * & & & & * & & * & & & * & & & 85 \\
\hline \multirow{2}{*}{$\begin{array}{l}\text { Red Eléctrica Corporación, } \\
\text { S.A. }\end{array}$} & 2015 & * & & & * & & & & * & & * & & & * & & & * & & & * & & & 85 \\
\hline & 2016 & * & & & * & & & & * & & * & & & * & & & * & & & * & & & 85 \\
\hline \multirow{2}{*}{ Repsol, S.A. } & 2015 & * & & & * & & & * & & & * & & & * & & & * & & & * & & & 100 \\
\hline & 2016 & * & & & * & & & * & & & * & & & * & & & * & & & * & & & 100 \\
\hline \multirow{2}{*}{$\begin{array}{l}\text { Siemens Gamesa Renewable } \\
\text { Energy, S.A. }\end{array}$} & 2015 & * & & & * & & & * & & & * & & & * & & & * & & & * & & & 100 \\
\hline & 2016 & * & & & * & & & * & & & & * & & * & & & * & & & * & & & 85 \\
\hline \multirow{2}{*}{ Técnicas Reunidas, S.A. } & 2015 & & * & & * & & & & * & & * & & & & * & & * & & & * & & & 57 \\
\hline & 2016 & & * & & * & & & * & & & * & & & & * & & * & & & * & & & 71 \\
\hline \multirow{2}{*}{ Telefónica, S.A. } & 2015 & * & & & * & & & * & & & * & & & * & & & * & & & * & & & 100 \\
\hline & 2016 & * & & & * & & & * & & & * & & & * & & & * & & & * & & & 100 \\
\hline \multirow{2}{*}{ Viscofan, S.A. } & 2015 & * & & & * & & & * & & & * & & & & * & & * & & & * & & & 85 \\
\hline & 2016 & * & & & * & & & * & & & * & & & & * & & * & & & * & & & 85 \\
\hline \multicolumn{24}{|c|}{$\begin{array}{l}\text { R6: publicación en web de informes para la junta; R12: interés social; R14: política de selección; R45: contenido de la política de control y gestión de } \\
\text { riesgos; R53: supervisión cumplimiento de reglas GC, códigos de conducta y RSC; R54: contenido mínimo de la política de RSC; R55: información } \\
\text { sobre RSC en informe de gestión o informe especial. } \\
\text { Fuente: elaboración propia en base a los IAGC de las empresas. }\end{array}$} \\
\hline
\end{tabular}


BOLETÍN ECONÓMICO DE INFORMACIÓN COMERCIAL ESPAÑOLA (BICE) ISSN 0214-8307

\section{SUSCRIPCIÓN ANUAL}

\begin{tabular}{|c|c|c|c|}
\hline & $\begin{array}{l}\text { ESPAÑA } \\
1 \text { año }\end{array}$ & $\begin{array}{c}\text { UNIÓN EUROPEA } \\
1 \text { año }\end{array}$ & $\begin{array}{c}\text { RESTO DEL } \\
\text { MUNDO } \\
1 \text { año }\end{array}$ \\
\hline SUSCRIPCIÓN & $65,00 €$ & $85,00 €$ & $85,00 €$ \\
\hline $\begin{array}{l}\text { Gastos de envío } \\
\text { España }\end{array}$ & $5,76 €$ & $24,36 €$ & $30,00 €$ \\
\hline $\begin{array}{l}\text { Más } 4 \% \text { de IVA. } \\
\text { Excepto Canarias, Ceuta y Melilla }\end{array}$ & $2,83 €$ & & \\
\hline TOTAL & $73,59 €$ & $109,36 €$ & $115,00 €$ \\
\hline
\end{tabular}

\section{EJEMPLARES SUELTOS}

\begin{tabular}{|c|c|c|c|}
\hline \multicolumn{4}{|c|}{ BOLETÍN ECONÓMICO DE INFORMACIÓN COMERCIAL ESPAÑOLA } \\
\hline & $\begin{array}{l}\text { ESPAÑA } \\
1 \text { ejemplar }\end{array}$ & $\begin{array}{l}\text { UNIÓN EUROPEA } \\
1 \text { ejemplar }\end{array}$ & $\begin{array}{c}\text { RESTO DEL } \\
\text { MUNDO } \\
1 \text { ejemplar }\end{array}$ \\
\hline NÚMERO SUELTO & $7,00 €$ & $9,00 €$ & $9,00 €$ \\
\hline $\begin{array}{l}\text { Gastos de envío } \\
\text { España }\end{array}$ & $0,48 €$ & $2,03 €$ & $2,50 €$ \\
\hline $\begin{array}{l}\text { Más } 4 \% \text { de IVA. } \\
\text { Excepto Canarias, Ceuta y Melilla }\end{array}$ & $0,30 €$ & & \\
\hline TOTAL & $7,78 €$ & $11,03 €$ & $11,50 €$ \\
\hline \multicolumn{4}{|c|}{ BOLETÍN ECONÓMICO DE INFORMACIÓN COMERCIAL ESPAÑOLA } \\
\hline & $\begin{array}{l}\text { ESPAÑA } \\
1 \text { ejemplar }\end{array}$ & $\begin{array}{l}\text { UNIÓN EUROPEA } \\
1 \text { ejemplar }\end{array}$ & $\begin{array}{l}\text { RESTO DEL } \\
\text { MUNDO } \\
1 \text { ejemplar }\end{array}$ \\
\hline $\begin{array}{l}\text { NÚMERO SUELTO } \\
\text { EXTRAORDINARIO }\end{array}$ & $12,00 €$ & $15,00 €$ & $15,00 €$ \\
\hline Gastos de envío España & $0,48 €$ & $2,03 €$ & $2,50 €$ \\
\hline $\begin{array}{l}\text { Más } 4 \% \text { de IVA. } \\
\text { Excepto Canarias, Ceuta y Melilla }\end{array}$ & $0,50 €$ & & \\
\hline TOTAL & $12,98 €$ & $17,03 €$ & $17,50 €$ \\
\hline
\end{tabular}

\section{DATOS}

Nombre y apellidos

Empresa

Domicilio

D.P.

N.I.F.

Teléf.

Email

\section{DATOS DEL EDITOR:}

NIF:S2800568D

Transferencia a la cuenta de ingresos por venta de publicaciones del Ministerio de Economía y Competitividad.

IBERCAJA. Calle Alcalá 29. 28014 MADRID (ESPAÑA) CÓDIGO CUENTA CLIENTE: 2085-9252-07-0330598330 CÓDIGO BIC DE IBERCAJA: CAZRES2Z

IBAN: ES47 2085-9252-07-0330598330

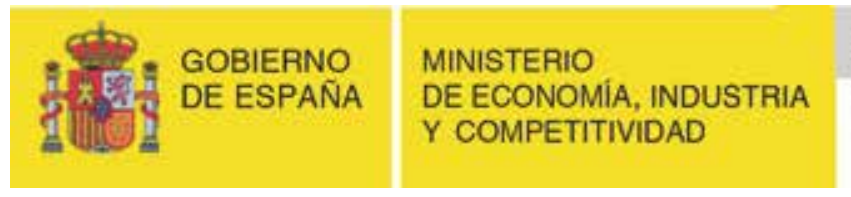




\section{NORMAS DE ESTILO DE PUBLICACIÓN}

La persona o personas interesadas en presentar un artículo para su publicación en el Boletín de Información Comercial Española (BICE) deberán enviar el artículo en formato Microsoft Word a la dirección de correo electrónico revistasice.sscc@comercio.mineco.es

El documento debe cumplir las siguientes características:

1. Ser material original no publicado ni presentado en otro medio de difusión.

2. La extensión total del trabajo (incluyendo cuadros, gráficos, tablas, notas, etcétera) no debe ser inferior a 15 páginas ni superior a 20. La fuente será Times New Roman, tamaño 12 y espaciado doble. Estar paginado en la parte inferior derecha de cada página.

3. En la primera página se hará constar el título del artículo que deberá ser breve, claro, preciso e informativo y la fecha de conclusión del mismo. Nombre y dos apellidos del autor o autores, filiación institucional, dirección, teléfono y correo electrónico de cada uno de ellos, así como la forma en que desean que sus datos aparezcan.

4. En la segunda página del texto se incluirá:

- El título.

- Un resumen del trabajo con una extensión máxima de 10 líneas con la siguiente estructura: objetivo, método y principal resultado o conclusión.

- De 2 a 6 palabras clave que no sean coincidentes con el título.

- De 1 a 5 códigos de materias del Journal of Economic Literature (clasificación JEL) para lo cual pueden acceder a la siguiente dirección electrónica:

https://www.aeaweb.org/jel/guide/jel.php

5. En las siguientes páginas se incluirán el texto, la información gráfica y la bibliografía con la siguiente organización.

- Se incluirá, por este orden, introducción, desarrollo, conclusiones y bibliografía y anexos si los hubiera. Los apartados y subapartados se numerarán en arábigos respondiendo a una sucesión continuada utilizando un punto para separar los niveles de división, según el siguiente modelo:

\section{Título del apartado}

\subsection{Título del apartado}

\subsubsection{Título del apartado}

\section{Título del apartado}

- Las notas de pie de página irán integradas en el texto y su contenido debe estar al final de su misma página en tamaño 10 y espacio sencillo.

- En un archivo Excel independiente se incluirá la representación gráfica (cuadros, gráficos, diagramas, figuras, etc.), que debe llevar título, estar numerada y referenciada en el texto.

En la parte inferior se incluirá la fuente de información y/o notas aclaratorias.

- Las citas de libros y artículos en el texto, se indicarán entre paréntesis con el apellido del autor y el año. Ej.: (Martínez, 1991).

- Las referencias a siglas deben ir acompañadas, en la primera ocasión en que se citen, de su significado completo.

- La bibliografía se ordenará alfabéticamente siguiendo las normas de la American Psychological Association (Harvard-APA): http://cibem.org/paginas/img/apa6.pdf

Libros

APELLIDOS, A.A. (año de publicación). Título del libro (edición) (volumen). Ciudad: Editorial.

Artículo en revista científica

APELLIDOS, A.A. (año de publicación). «Título del artículo». Título de la revista, volumen (número), números de páginas.

\section{Documento en línea}

APELLIDOS, A.A. u ORGANISMO (año, mes de publicación). Título, [en línea]. Ciudad: Editorial. Disponible en:

http://cenamb.rect.ucv.ve/siamaz/dicciona/canaima/canaima2.htm [Recuperado: 2000, 3 de junio]. 